\title{
GENERALIZATION OF THE BIG PICARD THEOREM
}

\author{
BY MYUNG HE KWACK ${ }^{1}$
}

Communicated by S. Smale, Feburary 29, 1968

S. Kobayashi defined a pseudodistance $d$ on a complex manifold in such a manner that it depends only on the complex structure of the complex manifold in question [7]. The definition of the pseudodistance can be extended word for word to a complex space (see [3] for definition of a complex space). Let $D$ be the open unit disk in the complex plane $\boldsymbol{C}$ and $\rho$ the Poincaré-Bergman metric of $D$. Given two points $p$ and $q$ of a complex space $X$, choose the following objects:

(1) points $p=p_{0}, p_{1}, \cdots, p_{k}=q$ of $X$, and

(2) points $a_{1}, \cdots, a_{k}, b_{1}, \cdots, b_{k}$ of $D$ and holomorphic mappings $f_{1}, \cdots, f_{k}$ from $D$ into $X$ such that $f_{i}\left(a_{i}\right)=p_{i-1}$ and $f_{i}\left(b_{i}\right)=p_{i}$ for $i=1, \cdots, k$. For each choice of points and mappings satisfying (1) and (2), consider the number $\rho\left(a_{1}, b_{1}\right)+\cdots+\rho\left(a_{k}, b_{k}\right)$. Let $d(p, q)$ be the infimum of the numbers obtained in this manner for all possible choices.

It is easy to verify that $d$ is a pseudodistance on $X$. We shall call a complex space hyperbolic if the pseudodistance $d_{X}$ is a distance. The concept of a hyperbolic space is a generalization of a Riemann surface of hyperbolic type in the sense that a Riemann surface of hyperbolic type is a hyperbolic space. A hyperbolic space $\left(X, d_{X}\right)$ is said to be complete if for any point $p$ of $X$ and any positive number $r$, the closed ball of radius $r$ around $p$ is compact.

The purpose of this paper is to generalize the big Picard theorem which states that a holomorphic mapping from the punctured disk into the Riemann sphere $P_{1}(C)$ minus three points can be extended to a holomorphic mapping from the whole disk into $P_{1}(C)$. H. Huber extended this theorem to the case where the image space is a domain $G$ of hyperbolic type in a Riemann surface $R$ such that the closure of $G$ in $R$ is compact [4].

THEOREM 1. Let $f$ be a holomorphic mapping from the punctured disk $D^{*}$ into a hyperbolic space $X$. Moreover, assume that the complex space $X$ is compact. Then $f$ can be extended to a holomorphic mapping from the whole disk into $X$.

1 This note is an abstract of the author's Ph.D dissertation written under the guidance of Professor S. Kobayashi. 
THEOREM 2. Let $M$ be a complex manifold and $A$ an analytic subset of $M$ of codimension at least 1 . Also let $X$ be a complete hyperbolic space which is compact $X$. Then a holomorphic mapping $f: M-A \rightarrow X$ can be extended to a unique holomorphic mapping from $M$ into $Y$. If, moreover, $A$ is of codimension at least 2 , the assumption that $X$ is compact can be dropped.

Theorem 3. Let $N$ be a bounded symmetric domain and $\Gamma$ an arithmetic group of transformations acting properly discontinuously on $N$. (It is known that the quotient space $N / \Gamma$ can be provided with a structure of complex space such that the projection $\pi: N \rightarrow N / \Gamma$ is holomorphic.) Let $Y$ be the compactification of $N / \Gamma$ by Borel and Baily [1]. If $f$ is a holomorphic mapping from the punctured disk $D^{*}$ into $N / \Gamma$ which can be lifted locally, then $f$ can be extended to a holomorphic mapping from the whole disk into $Y$. (We say that a holomorphic mapping $g$ from a complex space $X$ into $N / \Gamma$ can be lifted locally if given any point $p$ of $X$ there exist an open neighborhood $U$ of $p$ in $X$ and a holomorphic mapping $\tilde{g}_{U}: U \rightarrow N$ such that $\pi \circ \tilde{g}_{U}=g$ on $\left.U.\right)$

Theorem 4. Let $N, \Gamma$ and $Y$ be as in the previous theorem and moreover assume that $N$ is complete. If $A$ is an analytic subset of a complex manifold $M$ of codimension at least 1 , then a holomorphic mapping $f: M-A \rightarrow N / \Gamma$ which can be lifted locally can be extended to a unique holomorphic mapping from $M$ into $Y$. If, in addition, $A$ is of codimension at least 2 , the image of the extension of $f$ lies in $N / \Gamma$.

One example satisfying the condition of Theorem 4 is the generalized upper-half plane $H_{n}$ of degree $n$ and the generalized modular group $\Gamma_{n}$, which acts properly discontinuously on $H_{n}$. I. Satake has obtained a compactification of the quotient space $V_{n}=H_{n} / \Gamma_{n}$. This compactification of $V_{n}$ by I. Satake is shown to be an analytic space which is isomorphic to a normal projective variety [1].

\section{REFERENCES}

1. W. Baily, Jr. and A. Borel, A compactification of arithmetic quotients of bounded symmetric domains, Ann. of Math. 84 (1966), 442-528.

2. H. Grauert and H. Reckziegel, Hermitesche Metriken und Normale Familien holomorpher Abbildungen, Math. Z. 89 (1965), 108-125.

3. R. Gunning and H. Rossi, Analytic functions of several complex variables, Series in Modern Analysis, Prentice-Hall, Englewood Cliffs, N. J., 1965.

4. H. Huber, Über analytische Abbildungen Riemannscher Flächen in sich, Comment. Math. Helv. 27 (1953), 1-72.

5. S. Kobayashi, Invariant distances on complex manifold and holomorphic mappings, J. Math. Soc. Japan 19 (1967), 460-480. 
6. - Distance, holomorphic mappings and the Schwarz lemma, J. Math. Soc. Japan 19 (1967), 481-485.

7. - Intrinsic metrics on complex manifolds, Bull. Amer. Math. Soc. 73 (1967), 347-349.

University of CALIFornia, Berkely

\title{
A CHARACTERIZATION OF BANACH ALGEBRAS WITH APPROXIMATE UNIT
}

\author{
BY DONALD CURTIS TAYLOR
}

Communicated by R. C. Buck, February 13, 1968

1. Introduction. Let $L^{1}(R)$ denote the space of all complex valued functions on the real line $R$ which are integrable on $R$ in the sense of Lebesgue. It is well known that $L^{1}(R)$ forms a Banach algebra where the multiplication is defined by convolution; that is,

$$
f_{*} g(x)=\int_{R} f(t) g(x-t) d t
$$

and the norm of an element is defined by $\|f\|=\int|f(t)| d t$. In [4] Rudin showed that every function in $L^{1}(R)$ is the convolution of two other functions. In other words, every element of the convolution algebra $L^{1}(R)$ can be factored in $L^{1}(R)$, although this algebra lacks a unit. Subsequently, Cohen [1] observed that the essential ingredient in Rudin's argument is that $L^{1}(R)$ has an approximate unit in the sense of the following definition.

Definition. A Banach algebra $B$ is said to have an approximate unit if there exists a real number $C \geqq 1$ and a collection $\left\{e_{\lambda}: \lambda \in \Lambda\right\}$ of elements of $B$, where the index set $\Lambda$ is a directed set, such that the following two conditions are satisfied: $\left\|e_{\lambda}\right\| \leqq C$, for each $\lambda$, and $\lim e_{\lambda} x=\lim x e_{\lambda}=x$, for each $x \in B$. Cohen went on to prove that the factorization theorem still holds in any Banach algebra with approximate unit.

The results in this note stem from the observation that multiple factorization occurs in the sup-norm algebra $C_{0}(R)$, the space of all complex valued continuous functions on $R$ that vanish at infinity; that is, if $f_{1}, f_{2}, \cdots, f_{n}$ are functions in $C_{0}(R)$ and $\delta>0$, then there exist functions $g, h_{1}, h_{2}, \cdots, h_{n}$ in $C_{0}(R)$ such that

$$
f_{i}=g h_{i} \quad \text { and } \quad\left\|f_{i}-h_{i}\right\|<\delta \quad(i=1,2, \cdots, n) .
$$

